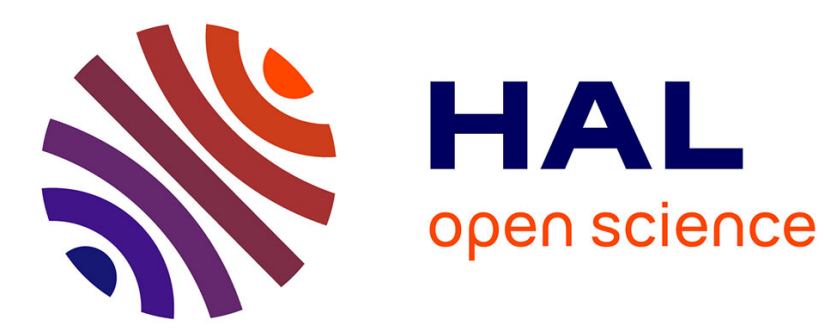

\title{
On the existence of Berge's strong equilibrium
}

Messaoud Deghdak, Monique Florenzano

\section{To cite this version:}

Messaoud Deghdak, Monique Florenzano. On the existence of Berge's strong equilibrium. 2011. halshs-00611851

\section{HAL Id: halshs-00611851 https://shs.hal.science/halshs-00611851}

Submitted on 27 Jul 2011

HAL is a multi-disciplinary open access archive for the deposit and dissemination of scientific research documents, whether they are published or not. The documents may come from teaching and research institutions in France or abroad, or from public or private research centers.
L'archive ouverte pluridisciplinaire HAL, est destinée au dépôt et à la diffusion de documents scientifiques de niveau recherche, publiés ou non, émanant des établissements d'enseignement et de recherche français ou étrangers, des laboratoires publics ou privés. 


\section{Documents de Travail du Centre d'Economie de la Sorbonne}

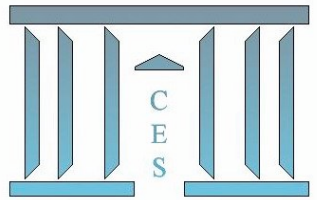

On the existence of Berge's strong equilibrium

Messaoud DeghDAK, Monique FlorenZANO

2011.39

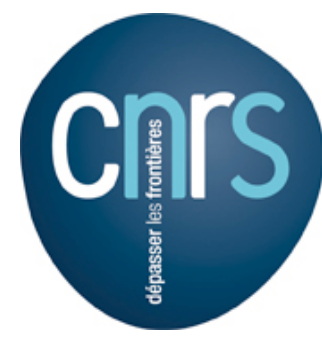




\title{
ON THE EXISTENCE OF BERGE'S STRONG EQUILIBRIUM
}

\author{
MESSAOUD DEGHDAK ${ }^{1}$ AND MONIQUE FLORENZANO ${ }^{2}$
}

\author{
${ }^{1}$ Laboratoire de Mathématiques Appliquées et Modélisation, Département de Mathématiques, \\ Université Mentouri, Constantine 25000, Algeria \\ deghdak@yahoo.fr \\ ${ }^{2}$ Centre d'Economie de la Sorbone, UMR 8174 CNRS-Université Paris 1 \\ 106-112, Boulevard de l'Hopital 75647, Paris CEDEX 13, France \\ monique.florenzano@univ-paris1.fr
}

\begin{abstract}
In this paper, we establish the existence of Berge's strong equilibrium for games with $\mathrm{n}$ persons in infinite dimensional strategy spaces in the case where the payoff function of each player is quasi-concave. Moreover, we study the continuity of Berge's strong equilibrium correspondence and prove that most of Berge's strong games are essential.
\end{abstract}

Keywords: Nash equilibrium; Strong Berge equilibrium; Fixed point; Essential games

AMS Subject Classification : $91 \mathrm{~A} 10$

\section{INTRODUCTION}

The concept of Nash equilibrium goes back to the paper of Nash (1951). For a noncooperative game, it is an equilibrium which is stable with respect to the deviation of any unique player. To get stronger stability properties, many concepts were introduced; let us quote for example the essential equilibrium (Wu and Jiang, 1962), the perfect equilibrium (Selten, 1975), and the proper equilibrium (Myerson, 1978). But already in 1957, on the basis of the notion of equilibrium for a partition $P$ with respect to the coalition $K$ of players, Berge (1957) defined the concept of Berge's strong equilibrium which insures a stronger stability than Nash's equilibrium. Indeed, a Berge strong equilibrium is stable with respect to the deviation of all players except one. Using the same idea, Zhukovskii (1994) introduced the concept of "Berge equilibrium in the sense of Zhukovskii" as an alternative solution for a noncooperative game when it has no Nash equilibrium or when it has several Nash equilibria. This equilibrium means that if each player plays his strategy at a Berge equilibrium, then he obtains the maximum payoff if all the remaining players play their strategy in the Berge equilibrium. It is worth noticing that Berge's strong equilibrium verifies stronger stability than the Aumann (1959) strong equilibrium and that the Berge equilibrium in the sense of Zhukovskii (1994) is totally different from the Nash 
equilibrium and the Berge strong equilibrium. For more details on Berge's strong equilibrium, see Krim (2001), Abalo and Kostreva (2004, 2005), Larbani et al (2007), Larbani and Nessah (2008).

The existence of Berge strong equilibrium and of essential equilibrium were respectively studied in Larbani and Nessah (2001) and in Wu and Jiang (1962). In their paper, Larbani and Nessah provide an existence result for the Berge equilibrium of a noncooperative game in normal form. The strategy space is a finite dimensional space and the payoff function of each person is continuous and concave. Wu and Jiang prove that every finite game can be closely approximated by an essential game. More precisely, let $x$ and $x^{\prime}$ be Nash equilibria of the noncooperative games $G$ and $G^{\prime}$ respectively. $x$ is said to be essential when the following condition is satisfied: if $G^{\prime}$ is "close" to $G$, then $x^{\prime}$ is "close" to $x$. $G$ is essential if all of its equilibria are essential. Note that this definition requires a topological structure on the spaces of games and issues. To this end, Wu and Jiang parametrize games by payoffs and issues as well. Then, using the upper semi-continuous property of the Nash equilibrium correspondence, they prove that most of the games are essential in the sense of Baire Category. Later, Yu (1999) extended the result found in Wu and Jiang (1962) not only to games defined in infinite dimensional spaces but also to other more general parametrizations of games such as: payoff profiles, payoff profiles and strategy sets, payoff profiles and feasible correspondences.

The aim of this paper is double.

First, we extend the existence result of Larbani and Nessah (2001) to infinite dimensional topological vector spaces, and we prove under weaker conditions on the payoff function the existence of Berge's strong equilibrium for noncooperative games in normal form and for abstract economies. With the local convexity of the strategy spaces, the proof is based on the Kakutani-Fan fixed point theorem. Without local convexity, the idea of the proof is inspired from $\mathrm{Yu}$ (1999) and consists in constructing a sequence of correspondences which verifies the Browder-Fan fixed point theorem (see Browder (1968), Theorem 1); then, passing to limit on the sequence of fixed points, we prove the existence of a Berge strong equilibrium.

Secondly, we prove, as in Yu, that most of Berge's strong equilibria of games are essential.

The paper is organized as follows. In Section 2, we define the Berge's strong equilibrium and give sufficient conditions for its existence. In Section 3, we prove the continuity of the Berge strong equilibrium correspondence. In Section 4, we study the essential Berge strong equilibria and games. Finally Section 5 is a short concluding section.

\section{Definition And existence of Berge's Strong EQUilibrium}

2.1. Berge's strong equilibrium of a noncooperative game. Let us consider the following noncooperative game in normal form:

$$
G=\left(I,\left(X_{i}, u_{i}\right)_{i \in I}\right)
$$


where $I=\{1, \cdots, n\}$ is a finite set of players, $X_{i}$ is the set of strategies of player $i$ and, if $X=\prod_{i=1}^{n} X_{i}$ denotes the set of issues of the game $G, u_{i}: X \rightarrow \mathbb{R}$ is the payoff function of $i$. As usual, we let $I \backslash\{i\}=\{1, \cdots, i-1, i+1, \cdots, n\}=\{j \in I: j \neq i\}$ and we denote $X_{-i}=\prod_{j \in I \backslash\{i\}} X_{j}$, and, if $x \in X=\prod_{i=1}^{n} X_{i}, x_{-i}=\left(x_{j}\right)_{j \neq i} \in X_{-i}$. Choosing a strategy $x_{i} \in X_{i}$, the aim of each player in the game $G$ is to maximize his payoff function.

Recall that $\bar{x} \in X$ is a Nash equilibrium of the game $G$ if for every $i \in I$, for all $x_{i} \in X_{i}$, $u_{i}(\bar{x}) \geq u_{i}\left(x_{i}, \bar{x}_{-i}\right)$. The following definition is due to Berge (1957).

Definition 2.1 (Berge (1957)). A Berge's strong equilibrium of the game $G$ is an n-tuple of strategies $\bar{x} \in X$ such that $\forall i \in I, \forall j \in I \backslash\{i\}, \forall y_{-i} \in X_{-i}, u_{j}(\bar{x}) \geq u_{j}\left(\bar{x}_{i,}, y_{-i}\right)$.

In other words, while at a Nash equilibrium no one of the players has interest to modify alone his strategy, at a Berge's strong equilibrium, for each player $i$, it is the complementary coalition which has no interest to deviate.

Remark 2.2. It is easily seen that a Berge's strong equilibrium is a Nash equilibrium and it is obvious that they coincide in games with two persons (see Larbani and Nessah (2001)).

In the following example, we show that a game may have Nash equilibria and no one Berge's strong equilibrium.

Example 2.3. Let us consider the following noncooperative game with three persons:

$$
\left(I,\left(X_{i}\right)_{i=1}^{3},\left(u_{i}\right)_{i=1}^{3}\right),
$$

where $X_{i}=[0,1]$ for each $i=1,2,3$ and $u_{1}(x)=x_{1}, u_{2}(x)=3 x_{1}^{3}-x_{3}, u_{3}(x)=x_{2}+x_{3}$. We prove by contraposition that there is no Berge's strong equilibrium. Indeed, if $\bar{x}$ is a Berge's strong equilibrium, we must have in particular for each $x_{2} \in X_{2}$ and $x_{3} \in X_{3}$,

$$
\begin{aligned}
& u_{2}(\bar{x}) \geq u_{2}\left(\bar{x}_{1}, x_{2}, x_{3}\right), \\
& u_{3}(\bar{x}) \geq u_{3}\left(\bar{x}_{1}, x_{2}, x_{3}\right) .
\end{aligned}
$$

Then, for every $\left(x_{2}, x_{3}\right) \in[0,1] \times[0,1], x_{3} \geq \bar{x}_{3}$ and $\bar{x}_{2}+\bar{x}_{3} \geq x_{2}+x_{3}$, which implies $\bar{x}_{3}=0$ and $\bar{x}_{2}+\bar{x}_{3} \geq 2$. We obtain a contradiction. However, any $x=(1, t, 1)$ where $t \in[0,1]$ is a Nash equilibrium.

Next, we give sufficient conditions for the existence of Berge's strong equilibrium. From now on, we assume that each strategy set $X_{i}$ is a subset of a (possibly infinite dimensional) Hausdorff, non necessarily locally convex, topological vector space $E_{i}$. For each $i$, let us denote by $u_{-i}$ the vector $\left(u_{j}\right)_{j \neq i}$ of utility functions of the members of $I \backslash\{i\}$ and call best reply correspondence for the complementary coalition $I \backslash\{i\}$ the correspondence $\Gamma_{-i}: X \rightarrow X$ defined by:

$$
\Gamma_{-i}(x)=\left\{y \in X: u_{-i}\left(x_{i}, y_{-i}\right) \geq u_{-i}\left(x_{i}, t_{-i}\right) \forall t_{-i} \in X_{-i}\right\} .
$$

For each $x \in X$, we set

$$
\Gamma(x)=\bigcap_{i \in I} \Gamma_{-i}(x)
$$


With these notations, a Berge's strong equilibrium is a fixed point of the correspondence $\Gamma$, that is an-tuple $\bar{x} \in \Gamma(\bar{x})$.

Theorem 2.4. Assume the following assumptions on $G$ :

A.1: For each $i \in I, X_{i}$ is a nonempty, convex and compact subset of $E_{i}$;

A.2: For each $i \in I, \forall j \in I \backslash\{i\}, \forall x_{i} \in X_{i}$, the function $y_{-i} \rightarrow u_{j}\left(x_{i}, y_{-i}\right)$ is quasi-concave on $X_{-i}$;

A.3: For each $i \in I, \forall j \in I \backslash\{i\}$, the function $u_{j}$ is upper semicontinuous on $X_{i} \times X_{-i}$;

A.4: For each $i \in I, \forall j \in I \backslash\{i\}, \forall x_{-i} \in X_{-i}$, the function $y_{i} \rightarrow u_{j}\left(y_{i}, x_{-i}\right)$ is lower semicontinuous on $X_{i}$;

A.5: $\forall x \in X, \Gamma(x) \neq \varnothing$.

Then, there exists a Berge's strong equilibrium.

Proof. Let us first assume that each strategy space $E_{i}$ is locally convex. We remark that, under the assumptions A.1 - A.4 of the theorem, each correspondence $\Gamma_{-i}$ is convex valued but may have empty values. Under these assumptions, the correspondence $\Gamma$ is closed ${ }^{1}$ (thus upper semicontinuous with compact values) and the set $F=\{x \in X: \Gamma(x) \neq \varnothing\}$ is closed in $X$. Indeed, it follows from assumptions A.1, A.3 and A.4 that for every $i \in I$ and for every $j \in I \backslash\{i\}$, the function:

$$
x_{i} \rightarrow \max _{t_{-i} \in X_{-i}} u_{j}\left(x_{i}, t_{-i}\right)
$$

is continuous (see $\mathrm{Yu}(1999)$, Lemma 2.4 ) or (see, Aubin (1984), Theorem 1, p. 67). The correspondence $x_{i} \rightarrow X_{-i}$ is obviously continuous and an easy adaptation of the proof of the Berge maximum theorem (see Aubin (1984), Theorem 3, p. 70, or Aliprantis and Border (1999), Theorem 16.31) shows that the correspondence

$$
x_{i} \rightarrow\left\{y: u_{j}\left(x_{i,} y_{-i}\right)=\max _{t_{-i} \in X_{-i}} u_{j}\left(x_{i}, t_{-i}\right)\right\}
$$

has a closed graph. As intersection of closed correspondences, each correspondence $\Gamma_{-i}$ is closed, thus upper semicontinuous with compact values. Consequently, if $x^{\nu} \rightarrow x$ with, for each $\nu$ of a directed set, $x^{\nu} \in F$, that is, $y^{\nu} \in \Gamma\left(x^{\nu}\right)$. In view of the compactness of $X$, one can assume without loss of generality that $y^{\nu} \rightarrow y$ and conclude that $y \in \Gamma(x)$ and $x \in F$. Adding now Assumption A.5, it follows from the Kakutani-Fan theorem that $\Gamma$ has a fixed point and the game $G$ has a Berge's strong equilibrium.

Without local convexity of the strategy spaces $E_{i}$, the idea of the proof of Theorem 2.4 is borrowed with some slight modifications from $\mathrm{Yu}$ (1999). Fix any integer $k$ and define the correspondence $W_{k}: X \rightarrow X$ as follows:

$$
W_{k}(x)=\left\{z \in X: \forall i \in I, \forall j \in I \backslash\{i\}, u_{j}\left(x_{i}, z_{-i}\right)>\max _{y_{-i} \in X} u_{j}\left(x_{i}, y_{-i}\right)-\frac{1}{k}\right\} .
$$

\footnotetext{
${ }^{1}$ For definition and properties of the different continuity concepts for correspondences, we refer the reader to Aliprantis and Border (1999), Aubin (1984) or to the Appendix of Florenzano (2003). For statements of classical fixed point theorems, we refer to Aliprantis and Border (1999), Florenzano (2003).
} 
It follows from the compactness of the sets $X_{i}$ and the assumptions A.3 and A.5 that each $W_{k}(x)$ is nonempty. Indeed, for every $i \in I$ and for every $j \in I \backslash\{i\}$, there exists $z^{j} \in X$ such that:

$$
u_{j}\left(x_{i}, z_{-i}^{j}\right)>\max _{y_{-i} \in X_{-i}} u_{j}\left(x_{i}, y_{-i}\right)-\frac{1}{k} .
$$

A fortiori, if $z \in \Gamma(x)$ then $z \in W_{k}(x)$. From assumption A.2, it follows that each $W_{k}(x)$ is convex. Using assumptions A.1, A.3 and A.4 and the Berge maximum theorem, it follows that for each $i \in I$ and for each $j \in I \backslash\{i\}$ the function:

$$
x_{i} \rightarrow \max _{y_{-i} \in X_{-i}} u_{j}\left(x_{i}, y_{-i}\right),
$$

is continuous on $X_{i}$. It then follows from the inequalities defining $W_{k}(x)$ in $(2.5)$ that $z \in W_{k}(t)$ still holds for every $t$ in some neighborhood of $x$. Applying the Browder-Fan theorem in Browder (1968), let $\bar{x}^{k}$ a fixed point of the correspondence $W_{k}$. Thus,

$$
\forall i \in I, \forall j \in I \backslash\{i\}, u_{j}\left(\bar{x}^{k}\right)=u_{j}\left(\bar{x}_{i}^{k}, \bar{x}_{-i}^{k}\right)>\max _{y_{-i} \in X_{-i}} u_{j}\left(\bar{x}_{i}^{k}, y_{-i}\right)-\frac{1}{k} .
$$

Since each $X_{i}$ is a compact, one can assume without loss of generality that the sequence $\left(\bar{x}^{k}\right)_{k}$ converges to $\bar{x} \in X$. Passing to limit in (2.7) and using assumption A.3 and once more the continuity of the function $\max y_{-i} \in X_{-i} u_{j}\left(x_{i,} y_{-i}\right)$, we get:

$$
\begin{aligned}
& \forall i \in I, \forall j \in I \backslash\{i\}, u_{j}\left(\bar{x}_{i}, \bar{x}_{-i}\right) \geq \lim _{k \rightarrow+\infty} \sup u_{j}\left(\bar{x}_{i}^{k}, \bar{x}_{-i}^{k}\right) \geq \\
& \lim _{k \rightarrow+\infty y_{-i} \in X_{-i}} u_{j}\left(\bar{x}_{i}^{k}, y_{-i}\right) .
\end{aligned}
$$

Since

$$
\lim _{k \rightarrow+\infty} \max _{y_{-i} \in X_{-i}} u_{j}\left(\bar{x}_{i}^{k}, y_{-i}\right)=\max _{y_{-i} \in X_{-i}} u_{j}\left(\bar{x}_{i}, y_{-i}\right)
$$

then

$$
\forall i \in I, \forall j \in I \backslash\{i\}, u_{j}\left(\bar{x}_{i}, \bar{x}_{-i}\right)=\max _{y_{-i} \in X_{-i}} u_{j}\left(\bar{x}_{i}, y_{-i}\right),
$$

and $\bar{x}$ is a Berge's strong equilibrium.

Remark 2.5. Theorem 2.4 extends to infinite dimensional strategy spaces as well as in several other respects Theorem 3.1 in Larbani and Nessah (2001). A cautious reader can verify that their demonstration, based on the Ky-Fan inequality (see Fan (1972)), can be adapted to the infinite dimensional framework and to our weakened continuity and concavity assumptions on payoff functions. Assumption A.5 in Theorem 2.4 is strong and means that for each strategy $x$, there exists another strategy such that, for this strategy, the complementary coalition has the best answer. One should note that assumption A.5 is not satisfied by the game of the Example 2.3. Moreover, as noticed by Larbani and Nessah (2001), this assumption is not necessary for the existence of a Berge's strong equilibrium. One can find in Larbani and Nessah (2001) an example of a game with Berge's strong equilibria that does not satisfy Assumption A.5. 
2.2. Berge's strong equilibrium of an abstract economy. We now consider the following generalized game that will be called abstract economy (see Borglin and Keiding (1976)):

$$
H=\left(I,\left(X_{i}, F_{i}, u_{i}\right)_{i \in I}\right),
$$

where $I=\{1, \cdots, n\}$ is a (finite) set of agents, $X_{i}$ is the strategy set of agent $i$, and if $X=\prod_{i \in I} X_{i}, u_{i}: X \rightarrow \mathbb{R}$ is the payoff function of $i$, while $F_{i}: X \rightarrow X_{i}$ denotes a feasibility correspondence for $i$ given the strategies of the other agents. The following definition extends Definition 2.1 to abstract economies.

Definition 2.6. A Berge's strong equilibrium of $H$ is a $n$-tuple of strategies, $\bar{x}$, such that for each $i \in I$,

(a) $\bar{x}_{i} \in F_{i}(\bar{x})$,

(b) $\forall j \in I \backslash\{i\}, \forall y_{-i} \in \prod_{j \in I \backslash\{i\}} F_{j}(\bar{x}), u_{j}(\bar{x}) \geq u_{j}\left(\bar{x}_{i}, y_{-i}\right)$.

The first condition guarantees that $\bar{x}$ is a vector of feasible strategies. If, as previously, we call, for each $i \in I$, best reply correspondence for the complementary coalition $I \backslash\{i\}$, the correspondence $\Gamma_{-i}: X \rightarrow X$ defined by:

$\Gamma_{-i}(x)=\left\{y \in X: y_{-i} \in \prod_{j \in I \backslash\{i\}} F_{j}(x)\right.$ and $\left.u_{-i}\left(x_{i}, y_{-i}\right) \geq u_{-i}\left(x_{i}, t_{-i}\right) \forall t_{-i} \in \prod_{j \in I \backslash\{i\}} F_{j}(x)\right\}$

both conditions (a) and (b) of Definition 2.6 jointly express that

$$
\bar{x} \in \bigcap_{i \in I} \Gamma_{-i}(\bar{x}) .
$$

Theorem 2.7. Assume the following assumptions on $H$ :

B.1: For each $i \in I, E_{i}$ is locally convex and $X_{i}$ is a nonempty, convex and compact subset of $E_{i}$;

B.2: For each $i \in I, \forall j \in I \backslash\{i\}, \forall x_{i} \in X_{i}$, the function $y_{-i} \rightarrow u_{j}\left(x_{i}, y_{-i}\right)$ is quasi-concave on $X_{-i}$;

B.3: For each $i \in I, \forall j \in I \backslash\{i\}$, the function $u_{j}$ is continuous on $X_{i} \times X_{-i}$;

B.4: For each $i \in I$, the correspondence $F_{i}: X \rightarrow X_{i}$ is continuous with nonempty, convex and compact values;

B.5: $\forall x \in X, \bigcap_{i \in I} \Gamma_{-i}(x) \neq \varnothing$.

Then $H$ has a Berge's strong equilibrium.

Proof. The proof is a slight modification of the proof given for Theorem 2.4 in the case where each strategy space is locally convex. For every $i \in I$, denote by $F_{-i}: X \rightarrow$ $\prod_{j \in I \backslash\{i\}} X_{j}$ the correspondence defined by:

$$
F_{-i}(x)=\prod_{j \in I \backslash\{i\}} F_{j}(x) .
$$


For every $j \in I \backslash\{i\}$, in view of the strengthened continuity assumptions on the functions $u_{j}$ and the continuity of the correspondence $F_{i}$, it follows from the maximum theorem that, for every $j \in I \backslash\{i\}$, the function:

$$
x_{i} \rightarrow \max _{t_{-i} \in F_{-i}(x)} u_{j}\left(x_{i}, t_{-i}\right)
$$

is continuous and the correspondence:

$$
x_{i} \rightarrow\left\{y: u_{j}\left(x_{i}, y_{-i}\right)=\max _{t_{-i} \in F_{-i}(x)} u_{j}\left(x_{i}, t_{-i}\right)\right\}
$$

has a closed graph. Once again, as intersection of closed correspondences, each correspondence $\Gamma_{-i}$ is closed, thus upper semicontinuous with compact values. The same is true for the correspondence $\Gamma: X \rightarrow X$ defined by $\Gamma(x)=\bigcap_{i \in I} \Gamma_{-i}(x)$. As by assumptions B.2 and B.5, $\Gamma$ is nonempty and convex valued, the existence of the Berge's strong equilibrium of $H$ follows from the Kakutani-Fan theorem.

\section{Continuity properties of Berge's strong equilibrium}

In this section, we consider games parametrized by payoff profiles and abstract economies parametrized by payoff profiles and feasibility correspondences and we prove that the Berge's strong equilibrium correspondence of this games is upper semicontinuous with nonempty and compact values.

3.1. Games parametrized by payoff profiles. Let us assume that, defined on the same strategy spaces, the games $\left(I,\left(X_{i}\right)_{i \in I},\left(u_{i}\right)_{i \in I}\right)$ are parametrized by the payoff profiles. More precisely, let $U$ be the set of payoff profiles $u=\left(u_{1}, \cdots, u_{n}\right)$ which satisfy the assumptions of Theorem 2.4 and verify $\sum_{i \in I} \sup _{x \in X}\left|u_{i}(x)\right|<\infty$, endowed with the distance $\rho$ defined as follows: For each $u^{1}=\left(u_{1}^{1}, \cdots, u_{n}^{1}\right)$ and $u^{2}=\left(u_{1}^{2}, \cdots, u_{n}^{2}\right) \in U$,

$$
\rho\left(u^{1}, u^{2}\right)=\sum_{i \in I} \sup _{x \in X}\left|u_{i}^{1}(x)-u_{i}^{2}(x)\right| .
$$

It is easily seen that $U$, endowed with the distance $\rho$, is a complete metric space (see, Appendix). Now, we define the Berge's strong equilibrium correspondence $F: U \rightarrow X$, where for each $u \in U, F(u) \subset X$ is the set of Berge's strong equilibria of the game. Then, we have the following theorem.

Theorem 3.1. For a noncooperative game, under the assumptions of Theorem 2.4, the Berge's strong equilibrium correspondence, $F$, is upper semicontinuous with nonempty and compact values.

Proof. It follows from Theorem 2.4 that the correspondence $F$ has nonempty values. We now prove that the graph of $F$ is closed. Let $\left(u^{\alpha}, x^{\alpha}\right)_{\alpha \in \Lambda}$ be a net of points of the graph of $F$ such that $\left(u^{\alpha}, x^{\alpha}\right) \rightarrow(u, x) \in U \times X$. Hence, $\forall \alpha \in \Lambda$,

$$
\forall i \in I, \forall j \in I \backslash\{i\}, u_{j}^{\alpha}\left(x_{i}^{\alpha}, x_{-i}^{\alpha}\right)=\max _{t_{-i} \in X_{-i}} u_{j}^{\alpha}\left(x_{i}^{\alpha}, t_{-i}\right) .
$$


We have, for each $i \in I$ and for each $j \in I \backslash\{i\}$,

$$
\begin{aligned}
& u_{j}\left(x_{i}, x_{-i}\right)-u_{j}^{\alpha}\left(x_{i}^{\alpha}, x_{-i}^{\alpha}\right)=u_{j}\left(x_{i}, x_{-i}\right)-u_{j}\left(x_{i}^{\alpha}, x_{-i}^{\alpha}\right) \\
& +u_{j}\left(x_{i}^{\alpha}, x_{-i}^{\alpha}\right)-u_{j}^{\alpha}\left(x_{i}^{\alpha}, x_{-i}^{\alpha}\right),
\end{aligned}
$$

and

$$
\begin{aligned}
& u_{j}\left(x_{i}, x_{-i}\right)-u_{j}\left(x_{i}^{\alpha}, x_{-i}^{\alpha}\right)+u_{j}\left(x_{i}^{\alpha}, x_{-i}^{\alpha}\right)-u_{j}^{\alpha}\left(x_{i}^{\alpha}, x_{-i}^{\alpha}\right) \geq \\
& u_{j}\left(x_{i}, x_{-i}\right)-u_{j}\left(x_{i}^{\alpha}, x_{-i}^{\alpha}\right)-\rho\left(u, u^{\alpha}\right) .
\end{aligned}
$$

From the upper semicontinuity of the function $u_{j}$, it follows that

$$
u_{j}\left(x_{i}, x_{-i}\right) \geq \lim _{\alpha} \sup u_{j}\left(x_{i}^{\alpha}, x_{-i}^{\alpha}\right) .
$$

From (3.5) and $\lim _{\alpha} \rho\left(u, u^{\alpha}\right)=0$, we deduce:

$$
u_{j}\left(x_{i}, x_{-i}\right)-\limsup _{\alpha} u_{j}^{\alpha}\left(x_{i}^{\alpha}, x_{-i}^{\alpha}\right) \geq 0 .
$$

On the other hand, let $\varepsilon>0$ and for each $i \in I, j \in I \backslash\{i\}$, take $y_{-i} \in X_{-i}$ such that:

$$
u_{j}\left(x_{i}, y_{-i}\right)>\max _{t_{-i} \in X_{-i}} u_{j}\left(x_{i}, t_{-i}\right)-\varepsilon \text {. }
$$

Since $\rho\left(u, u^{\alpha}\right) \rightarrow 0, x_{i}^{\alpha} \rightarrow x_{i}$ and $u_{j}$ is lower semicontinuous, there exists $\alpha_{0} \in \Lambda$ with $\rho\left(u, u^{\alpha}\right)<\varepsilon$ and $u_{j}\left(x_{i}^{\alpha}, y_{-i}\right)>u_{j}\left(x_{i}, y_{-i}\right)-\varepsilon$ for all $\alpha \geq \alpha_{0}$. From (3.2), we deduce:

$$
\begin{aligned}
& u_{j}^{\alpha}\left(x_{i}^{\alpha}, x_{-i}^{\alpha}\right) \geq u_{j}^{\alpha}\left(x_{i}^{\alpha}, y_{-i}\right) \geq u_{j}\left(x_{i}^{\alpha}, y_{-i}\right)-\rho\left(u, u^{\alpha}\right) \geq \\
& u_{j}\left(x_{i}^{\alpha}, y_{-i}\right)-\varepsilon \geq u_{j}\left(x_{i}, y_{-i}\right)-2 \varepsilon .
\end{aligned}
$$

Hence,

$$
u_{j}^{\alpha}\left(x_{i}^{\alpha}, x_{-i}^{\alpha}\right)>\max _{t_{-i} \in X_{-i}} u_{j}\left(x_{i}, t_{-i}\right)-3 \varepsilon
$$

From (3.6), we obtain:

$$
u_{j}\left(x_{i}, x_{-i}\right) \geq \lim _{\alpha} \sup u_{j}^{\alpha}\left(x_{i}^{\alpha}, x_{-i}^{\alpha}\right) \geq \liminf _{\alpha} u_{j}^{\alpha}\left(x_{i}^{\alpha}, x_{-i}^{\alpha}\right) \geq \max _{t_{-i} \in X_{-i}} u_{j}\left(x_{i}, t_{-i}\right)-3 \varepsilon .
$$

When $\varepsilon \rightarrow 0$, we have $u_{j}\left(x_{i}, x_{-i}\right)=\max _{t_{-i} \in X_{-i}} u_{j}\left(x_{i}, t_{-i}\right)$. As this is true for each $i \in I$ and for each $j \in I \backslash\{i\}, x \in F(u)$ and the graph of $F$ is closed. Then $F$ has closed values in the compact set $X$ and is upper semicontinuous on $U$.

3.2. Abstract economies parametrized by payoff profiles and feasibility correspondences. In this subsection, we assume (strong assumption) that each strategy space $E_{i}$ is a normed space. Let us consider the generalized game $\left(I,\left(F_{i}\right)_{i \in I},\left(u_{i}\right)_{i \in I}\right)$ parametrized by payoff profiles and the feasibility strategy correspondences. More precisely, we denote by $V$ the set of vectors $v=\left(\left(u_{1}, \cdots, u_{n}\right),\left(F_{1}, \cdots, F_{n}\right)\right)$ where $\left(u_{1}, \cdots, u_{n}\right)$ is the payoff profile and $\left(F_{1}, \cdots, F_{n}\right)$ are feasibility correspondences which satisfy the conditions of Theorem 2.7 . 
For each $v^{1}=\left(u_{1}^{1}, \cdots, u_{n}^{1}, F_{1}^{1}, \cdots, F_{n}^{1}\right) \in V, v^{2}=\left(u_{1}^{2}, \cdots, u_{n}^{2}, F_{1}^{2}, \cdots, F_{n}^{2}\right) \in V$, define as in $\mathrm{Yu}$ (1999), p. 369, with slight modifications, the function $\rho: V \rightarrow \overline{\mathbb{R}}$

$$
\rho\left(v^{1}, v^{2}\right)=\begin{gathered}
\sum_{i=1}^{n} \sup _{x \in X}\left|u_{i}^{1}(x)-u_{i}^{2}(x)\right|+\sum_{i=1}^{n} \sup _{x \in X} H_{i}\left(F_{i}^{1}(x), F_{i}^{2}(x)\right) \\
+\sum_{i=1}^{n} \sup _{x \in X} T_{i}\left(\prod_{j \in I \backslash\{i\}} F_{j}^{1}(x), \prod_{j \in I \backslash\{i\}} F_{j}^{2}(x)\right)
\end{gathered}
$$

where for each $i \in I$ and for each $j \in I \backslash\{i\}, H_{i}$ is the Hausdorff distance on the set of subsets of the normed strategy space $E_{i}$ and $T_{i}$ is the Hausdorff distance on the set of subsets of the product normed space $\prod_{j \in I \backslash\{i\}} E_{j}$. Suppose that $\rho$ is well defined (i.e. has finite values); then $V$ is a complete metric space. Now, we define the Berge's strong equilibria correspondence $J: V \rightarrow X$, where for each $v \in V, J(v) \subset X$ is the set of Berge's strong equilibria of the game. Then, we have the following theorem.

Theorem 3.2. For an abstract economy defined on normed strategy spaces, under the assumptions of Theorem 2.7, the Berge's strong equilibrium correspondence $J$ is upper semicontinuous with nonempty and compact values.

Proof. The correspondence $\mathrm{J}$ has nonempty values by Theorem 2.7. We now prove that the graph of $J$ is closed. Let $\left(v^{\alpha}, x^{\alpha}\right)_{\alpha \in \Lambda}$ be a net of the graph of $J$ such that $\left(v^{\alpha}, x^{\alpha}\right) \rightarrow$ $(v, x) \in V \times X$. Then for each $\alpha \in \Lambda$, we have :

$$
\forall i \in I, \forall j \in I \backslash\{i\}, x_{i}^{\alpha} \in F_{i}^{\alpha}\left(x^{\alpha}\right),
$$

and

$$
u_{j}^{\alpha}\left(x_{i}^{\alpha}, x_{-i}^{\alpha}\right)=\max \left\{u_{j}^{\alpha}\left(x_{i}^{\alpha}, t_{-i}\right): t_{-i} \in \prod_{j \in I \backslash\{i\}} F_{j}^{\alpha}\left(x^{\alpha}\right)\right\} .
$$

Let $M$ be any subset of a topological space $S$ and denote by $K(M)$ the set of convex subsets of $M$ endowed with the Vietoris topology (see Engelking (1968), p163). Recall that if $S$ is a metric space, then the Vietoris topology on $K(M)$ coincides with the Hausdorff topology induced by $S$ on the compact subsets of $M$ (see Klein and Thompson (1984), Corollary 4.2.3, p 41). We have:

$$
d_{i}\left(x_{i}, F_{i}(x)\right) \leq \begin{gathered}
d_{i}\left(x_{i}, x_{i}^{\alpha}\right)+d_{i}\left(x_{i}^{\alpha}, F_{i}^{\alpha}\left(x^{\alpha}\right)\right)+ \\
H_{i}\left(F_{i}^{\alpha}\left(x^{\alpha}\right), F_{i}\left(x^{\alpha}\right)\right)+H_{i}\left(F_{i}\left(x^{\alpha}\right), F_{i}(x)\right) .
\end{gathered}
$$

From (3.14), we obtain:

$$
d_{i}\left(x_{i}, F_{i}(x)\right) \leq d_{i}\left(x_{i}, x_{i}^{\alpha}\right)+\rho\left(v^{\alpha}, v\right)+H_{i}\left(F_{i}\left(x^{\alpha}\right), F_{i}(x)\right) .
$$

Since for each $i \in I$, the correspondence $F_{i}: X \rightarrow X_{i}$ has compact values and is continuous, $F_{i}: X \rightarrow K\left(X_{i}\right)$ is a continuous function and

$$
\lim _{\alpha} H_{i}\left(F_{i}\left(x^{\alpha}\right), F_{i}(x)\right)=0 .
$$

Passing to limit in (3.15), we obtain $d_{i}\left(x_{i}, F_{i}(x)\right)=0$ and then $x_{i} \in \overline{F_{i}(x)}$. But $F_{i}(x)$ is compact, thus for each $i \in I, x_{i} \in F_{i}(x)$. For each $i \in I$ and for each $j \in I \backslash\{i\}$, we have:

$$
\left|u_{j}^{\alpha}\left(x_{i}^{\alpha}, x_{-i}^{\alpha}\right)-u_{j}\left(x_{i}, x_{-i}\right)\right| \leq \rho\left(v^{\alpha}, v\right)+\left|u_{j}\left(x_{i}^{\alpha}, x_{-i}^{\alpha}\right)-u_{j}\left(x_{i}, x_{-i}\right)\right| .
$$


For $\alpha$ large enough, we get:

$$
\lim _{\alpha} u_{j}^{\alpha}\left(x_{i}^{\alpha}, x_{-i}^{\alpha}\right)=u_{j}\left(x_{i}, x_{-i}\right) .
$$

Since for each $i \in I$ and for each $j \in I \backslash\{i\}$, the correspondence $\prod_{j \in I \backslash\{i\}} F_{j}(x)$ is continuous, then

$$
\lim _{\alpha} T_{i}\left(\prod_{j \in I \backslash\{i\}} F_{j}\left(x^{\alpha}\right), \prod_{j \in I \backslash\{i\}} F_{j}(x)\right)=0 .
$$

From the definition of the distance $\rho$, we have:

$$
T_{i}\left(\prod_{j \in I \backslash\{i\}} F_{j}^{\alpha}\left(x^{\alpha}\right), \prod_{j \in I \backslash\{i\}} F_{j}\left(x^{\alpha}\right)\right) \leq \rho\left(v^{\alpha}, v\right) .
$$

Since

$$
\begin{aligned}
& T_{i}\left(\prod_{j \in I \backslash\{i\}} F_{j}^{\alpha}\left(x^{\alpha}\right), \prod_{j \in I \backslash\{i\}} F_{j}(x)\right) \leq T_{i}\left(\prod_{j \in I \backslash\{i\}} F_{j}^{\alpha}\left(x^{\alpha}\right), \prod_{j \in I \backslash\{i\}} F_{j}\left(x^{\alpha}\right)\right)+ \\
& T_{i}\left(\prod_{j \in I \backslash\{i\}} F_{j}\left(x^{\alpha}\right), \prod_{j \in I \backslash\{i\}} F_{j}(x)\right),
\end{aligned}
$$

then for $\alpha$ large enough, we have:

$$
\forall i \in I, \forall j \in I \backslash\{i\}, \lim _{\alpha} T_{i}\left(\prod_{j \in I \backslash\{i\}} F_{j}^{\alpha}\left(x^{\alpha}\right), \prod_{j \in I \backslash\{i\}} F_{j}(x)\right)=0 .
$$

For each $i \in I$ and for each $j \in I \backslash\{i\}, x_{i}^{\alpha}$ converges to $x_{i}$ and

$$
\lim _{\alpha} \sup _{\left(x_{i}, t\right) \in X_{i} \times X_{-i}}\left|u_{j}^{\alpha}\left(x_{i}, t\right)-u_{j}\left(x_{i}, t\right)\right|=0 .
$$

Then by Lemma 2.5 in $\mathrm{Yu}$ (1999), we obtain:

$$
\lim _{\alpha} \max _{t_{-i} \in \prod_{j \in I \backslash\{i\}} F_{j}^{\alpha}\left(x^{\alpha}\right)} u_{j}^{\alpha}\left(x_{i}^{\alpha}, t_{-i}\right)=\max _{t_{-i} \in \prod_{j \in I \backslash\{i\}} F_{j}(x)} u_{j}\left(x_{i}, t_{-i}\right) .
$$

Using (3.13) and (3.18), we get:

$$
\forall i \in I, \forall j \in I \backslash\{i\}, u_{j}\left(x_{i}, x_{-i}\right)=\max _{t_{-i} \in \prod_{j \in I \backslash\{i\}} F_{j}(x)} u_{j}\left(x_{i}, t_{-i}\right) .
$$

It follows that for each $i \in I$ and for each $j \in I \backslash\{i\}, x \in J(v)$ and the graph of $J$ is closed. Then $J$ has closed values in the compact set $X$ and is upper semicontinuous.

In the particular case, where for each $i \in I, F_{i}: X \rightarrow C K\left(X_{i}\right)$ is a constant function with values in $C K\left(X_{i}\right)$, where $C K\left(X_{i}\right) \subset K\left(X_{i}\right)$ denotes the set of compact and convex subsets of $X_{i}$, we find the model(B) of the games parametrized by the payoff profiles and the strategies sets (see Yu (1999), p.367). Then, under weaker conditions on $X_{i}$ and the strategy space $E_{i}$, we have the following theorem for games parametrized by strategy profiles and strategy sets. 
Theorem 3.3. Under Assumptions B.2-B.5 in Theorem 2.7 and if for each $i \in I, X_{i} \subset$ $E_{i}$ is closed and $E_{i}$ is a Hausdorff topological vector space, the Berge's strong equilibrium correspondence $J$ is upper semicontinuous with nonempty and compact values.

Proof. Let us consider the following noncooperative games $\left(I,\left(S_{i}\right)_{i=1}^{n},\left(u_{i}\right)_{i=1}^{n}\right)$ parametrized by payoff profiles and strategy sets. From Theorem $2.4, J$ has nonempty values. Let $x^{\alpha} \in J(v)$ a net where $v=\left(u_{1}, \cdots, u_{n}, S_{1}, \cdots, S_{n}\right) \in V$ and $\lim _{\alpha} x^{\alpha}=x$. Then, for each $i \in I$ and for each $j \in I \backslash\{i\}, u_{j}\left(x_{i}^{\alpha}, x_{-i}^{\alpha}\right)=\max _{t_{-i} \in S_{-i}} u_{j}\left(x_{i}^{\alpha}, t_{-i}\right)$. It follows from the continuity of $u_{i}$ and the maximum function, $\max _{t_{-i} \in S_{-i}} u_{j}\left(x_{i}, t_{-i}\right)=u_{j}\left(x_{i}, x_{-i}\right)$. Hence, $J$ has closed values in the compact set $\prod_{i=1}^{n} S_{i}$. Now, we prove that $J$ is upper semicontinuous. If it is not true at a point $y \in V$, then there exists an open set $O$ of $X$ and a net $v^{\alpha} \in V$ such that $O \supset J(y), \lim _{\alpha} v^{\alpha}=v, x^{\alpha} \in J\left(v^{\alpha}\right)$ and $x^{\alpha} \notin O$. Thus, $\lim _{\alpha} \rho\left(u^{\alpha}, u\right)=0$ and for each $i \in I, \lim _{\alpha} S_{i}^{\alpha}=S_{i}$ for the Vietoris topology on $C K\left(X_{i}\right)$. In view of Lemma 2.3 in $\mathrm{Yu}(1999)$, let $x$ be a cluster point of $x^{\alpha}$. It is obvious that $x \notin O \supset J(y)$ and hence there exists $i_{0} \in I, j_{0} \in I \backslash\left\{i_{0}\right\}$ and $\varepsilon_{0}>0$ such that:

$$
u_{j_{0}}\left(x_{i_{0}}, x_{-i_{0}}\right)<\max _{t_{-i_{0}} \in S_{-i_{0}}} u_{j_{0}}\left(x_{i_{0}}, t_{-i_{0}}\right)-\varepsilon_{0}=u_{j_{0}}\left(x_{i_{0}}, u_{-i_{0}}^{0}\right)-\varepsilon_{0}
$$

where $u_{-i_{0}}^{0} \in S_{-i_{0}}$. Let $X$ be a topological space and $t \in X$, we denote by $O(t)$ any open set of $X$ which contains a point $t$. By the continuity of $u_{j_{0}}$ at points $\left(x_{i_{0}}, x_{-i_{0}}\right),\left(x_{i_{0}}, u_{-i_{0}}^{0}\right)$ we have:

$$
\forall x^{\prime} \in O\left(x_{i_{0}}\right) \times O\left(x_{-i_{0}}\right), u_{j_{0}}\left(x_{i_{0}}^{\prime}, x_{-i_{0}}^{\prime}\right)-\varepsilon_{0} / 4<u_{j_{0}}\left(x_{i_{0}}, x_{-i_{0}}\right)
$$

and

$$
\forall x^{\prime} \in O\left(x_{i_{0}}\right) \times O\left(u_{-i_{0}}^{0}\right), u_{j_{0}}\left(x_{i_{0}}, u_{-i_{0}}^{0}\right)<u_{j_{0}}\left(x_{i_{0}}^{\prime}, x_{-i_{0}}^{\prime}\right)+\varepsilon_{0} / 4 .
$$

For each $j \in I \backslash\left\{i_{0}\right\}$, let $V_{j}\left(u_{j}^{0}\right) \subset X_{j}$ an open set such that $u_{j}^{0} \in S_{j}$ and $\prod_{j \in I \backslash\left\{i_{0}\right\}} V_{j}\left(u_{j}^{0}\right) \subset$ $O\left(u_{-i_{0}}^{0}\right)$. Since $V_{j}\left(u_{j}^{0}\right) \cap S_{j} \neq \varnothing$ then for $\alpha$ large enough, $\rho\left(u^{\alpha}, u\right)<\varepsilon_{0} / 4, V_{j}\left(u_{j}^{0}\right) \cap S_{j}^{\alpha} \neq \varnothing$ and $x^{\alpha} \in O\left(x_{i_{0}}\right) \times O\left(x_{-i_{0}}\right)$. Take $u_{-i_{0}}^{\alpha} \in O\left(u_{-i_{0}}^{0}\right) \cap \prod_{j \in I \backslash\left\{i_{0}\right\}} S_{j}^{\alpha}$. It is obvious that

$$
u_{j_{0}}^{\alpha}\left(x_{i_{0}}^{\alpha}, x_{-i_{0}}^{\alpha}\right)<u_{j_{0}}\left(x_{i_{0}}^{\alpha}, x_{-i_{0}}^{\alpha}\right)+\frac{\varepsilon_{0}}{4}<u_{j_{0}}\left(x_{i_{0}}, x_{-i_{0}}\right)+\frac{\varepsilon_{0}}{2} .
$$

From (3.26) we get:

$$
u_{j_{0}}^{\alpha}\left(x_{i_{0}}^{\alpha}, x_{-i_{0}}^{\alpha}\right)<u_{j_{0}}^{\alpha}\left(x_{i_{0}}^{\alpha}, u_{-i_{0}}^{\alpha}\right) .
$$

Since $u_{-i_{0}}^{\alpha} \in S_{-i_{0}}^{\alpha}$, we obtain a contradiction with the assumption that $x^{\alpha}$ is a net of Berge's strong equilibria. 


\section{Essential EqUiLibrium AND Games}

In this section, as in $\mathrm{Yu}$ (1999), we prove that most (in the sense of Baire Category) of games defined in subsections 3.1 and 3.2 are essential. Let $Y$ be any one of spaces $U, V$ and $T$ any one of the correspondences $F, J$ (see subsections 3.1 and 3.2). Recall that the Berge's strong equilibrium correspondence $T$ is upper semicontinuous and with nonempty and compact values (see Theorems 3.1 and 3.2). For each $x \in X$ and for each $y \in Y$, we denote by $\mathcal{V}(x)$ the set of neighborhood of $x$ and by $\mathcal{W}(y)$ the set of neighborhood of $y \in Y$. Now, we give a definition of essential games.

Definition 4.1. ( $\mathrm{Yu}(1999))$. Let $M$ be a nonempty and closed subset of $Y$ and $y \in M$. An element $x \in T(y)$ is called essential equilibrium of the game $y \in Y$ relative to $M$ if for any $O \in \mathcal{V}(x)$, there exists $W \in \mathcal{W}(y)$ such that for each $y_{1} \in M \cap W$, there exists $x_{1} \in T\left(y_{1}\right)$ with $x_{1} \in O$.

It follows from this definition that the game $y \in M$ is essential if and only if the correspondence $T: M \rightarrow K(X)$ is lower semicontinuous at $y$ (see Yu (1999), Theorem 4.1). In the following theorem, we prove that most of games $y \in Y$ are essential.

Theorem 4.2. Assume that for each $i \in I$, the strategy space $E_{i}$ is a normed space. Then most of games in subsections 3.1 and 3.2 are essential.

Proof. Let $M$ be a closed subset of $Y$ and consider the corresponcence $T: M \rightarrow K(X)$. Since $Y$ is a complete metric space then $M$ is a complete metric space. Thus $M$ is a Baire space. Also, as a subset of $\prod_{i \in I} E_{i}, X$ is a normed space. Since $T$ is upper semicontinuous, then applying Lemma 2.1 in $\mathrm{Yu}$ (1999), there exists a dense residual or a negligible subset $Q$ ( contains a countable intersection of open dense subsets) of $M$ such that $T$ is lower semicontinuous on $Q$. Thus, most of games in subsections 3.1 and 3.2 are essential.

If $X_{i}$ is closed in a Banach space $E_{i}$, we have the following theorem.

Theorem 4.3. Most of games parametrized by payoff profiles and strategies sets are essential.

Proof. Let $M$ be a closed subset of $V$. Since $X_{i}$ is closed in a Banach space $E_{i}$, then $X_{i}$ is a complete metric space. Applying Theorem 4.3.9 in Klein and Thompson (1984), $K\left(X_{i}\right)$ is a complete metric space. Since $C K\left(X_{i}\right) \subset K\left(X_{i}\right)$ is closed (see Yu (1999), Lemma 2.2), then $C K\left(X_{i}\right)$ is a complete metric space and $V=U \times \prod_{i \in I} C K\left(X_{i}\right)$ is a complete metric space. Then the conclusion of Theorem 4.3 follows from Theorem 3.3.

Remark 4.4. As noticed in Theorem 4.3 (see, Yu (1999)), all games in $Y$ which have one Berge's strong equilibrium are essential.

In the following example, inspired by Example 1 (see Zhou and Xiang (2007)), we show that not all games in subsection 3.1 are essential. 
Example 4.5. In this example, we consider a game in normal form with two persons where the payoff function of each person is defined on the subset $[-1,1] \times[0,1]$ as follows:

$$
u_{1}\left(x_{1}, x_{2}\right)=\left|x_{1}\right|, \quad u_{2}\left(x_{1}, x_{2}\right)=x_{2} .
$$

It is easily seen that the set of Berge's strong equilibria of this game is the set $\{(-1,1),(1,1)\}$. Let us consider the sequence of payoff functions of each person defined on $[-1,1] \times[0,1]$ by :

$$
u_{1}^{n}\left(x_{1}, x_{2}\right)=\left\{\begin{array}{cc}
l-x_{1} & \left(x_{1}, x_{2}\right) \in[-1,0] \times[0,1] \\
\left(1-\frac{1}{n}\right) x_{1} & \left(x_{1}, x_{2}\right) \in[0,1] \times[0,1]
\end{array}\right.
$$

and

$$
u_{2}^{n}\left(x_{1}, x_{2}\right)=\left(1-\frac{1}{n}\right) x_{2} .
$$

It is obvious that $u_{1}^{n}$ and $u_{2}^{n}$ are continuous functions and that the set of Berge's strong equilibria of the sequence $\left(u_{1}^{n}, u_{2}^{n}\right)$ is the set $\{(-1,1)\}$. Since $\left(u_{1}^{n}, u_{2}^{n}\right)$ converges for the distance $\rho$ (see, subsection 3.1) to $\left(u_{1}, u_{2}\right)$, then the Berge's strong equilibrium point $(1,1)$ is not essential. Similarly, the Berge's strong equilibrium point $(-1,1)$ is not essential. Indeed, it suffices to take:

$$
u_{1}^{n}\left(x_{1}, x_{2}\right)=\left\{\begin{array}{cc}
l-x_{1} & \left(x_{1}, x_{2}\right) \in[-1,0] \times[0,1] \\
\left(1+\frac{1}{n}\right) x_{1} & \left.\left(x_{1}, x_{2}\right) \in[0,1] \times 0,1\right]
\end{array}\right.
$$

and

$$
u_{2}^{n}\left(x_{1}, x_{2}\right)=\left(1+\frac{1}{n}\right) x_{2} .
$$

Remark 4.6. As in Theorem 2 in Zhou and Xiang (2007), we can see that all games in subsection 3.1 have a minimal essential set.

\section{Conclusion}

In this paper, we have given in Theorem 2.4 and Theorem 2.7 sufficient conditions of existence for Berge's strong equilibrium of cardinal noncooperative games and abstract economies, i.e., of games and abstract economies where players' preferences are represented by payoff functions. The conditions A.1 - A.4 of Theorem 2.4 (resp. conditions B.1 B.4 of Theorem 2.7) are standard but condition A.5 (resp. B.5) is difficult to verify. Theorem 2.4 extends the result of Larbani and Nessah (2001) to infinite dimensional strategy spaces. Furthermore, as in Yu (1999) for the Nash games, we have shown through Theorems 4.2 and 4.3 that most of Berge's strong games and abstract economies are essential in the sense of Baire Category. That is, there are cases for which Berge's strong games are not essential. Example 2 illustrates one of these cases. In future work, we will address the equilibrium existence problem for ordinal Berge strong games and abstract economies, i.e., for games where players' preferences do not admit payoff representations. We will also try to get equilibrium existence under more flexible conditions than A.5 of Theorem 2.4 and B.5 of Theorem 2.7. 


\section{APPENDIX}

Proposition 5.1. The set $U$ endowed with the distance $\rho$ is a complete metric space.

Proof. Let $u^{p}=\left(u_{1}^{p} \cdots, u_{n}^{p}\right) \in U$ be a Cauchy sequence. Let us prove that $u^{p}$ converges to $u=\left(u_{1}, \cdots, u_{n}\right) \in U$. By the definition of the set $U$ and $\rho$, we have for each $i=1, \cdots, n$ and for each $m \in \mathbb{N}, u_{i}^{p}$ is a bounded function on $X$ and

$$
\begin{aligned}
& \forall i=1, \cdots, n, \forall \epsilon>0, \exists n_{0}(\epsilon) \in \mathbb{N}: \forall m \geq n_{0}(\epsilon), \forall q \geq n_{0}(\epsilon) \Rightarrow \\
& \sup _{x \in X}\left|u_{i}^{m}(x)-u_{i}^{q}(x)\right| \leq \epsilon .
\end{aligned}
$$

Let $B(X)$ be the complete vector space of all bounded real functions on $X$ endowed with the uniform metric. Then from (5.1), $u_{i}^{m}$ converges to the function $u_{i} \in B(X)$. It follows:

$$
\forall i=1, \cdots, n, \forall \epsilon>0, \exists n_{0}(\epsilon) \in \mathbb{N}, \forall m \geq n_{0}(\epsilon) \Rightarrow \sup _{x \in X}\left|u_{i}^{m}(x)-u_{i}(x)\right| \leq \epsilon .
$$

Set $u=\left(u_{1}, \cdots, u_{n}\right)$. Then $\sum_{i=1}^{n} \sup _{x \in X}\left|u_{i}(x)\right|<\infty$. It is obvious that $u_{i}$ is quasi-concave (as uniform limit of quasi-concave functions $u_{i}^{m}$ ). Now, we show that $u_{i}$ is upper semicontinuous on $X$. Let $x_{\alpha}$ a net in $X$ such that $x_{\alpha}$ converges to $x$. Since

$$
u_{i}(x)-u_{i}\left(x_{\alpha}\right)=u_{i}(x)-u_{i}^{m}(x)+u_{i}^{m}(x)-u_{i}^{m}\left(x_{\alpha}\right)+u_{i}^{m}\left(x_{\alpha}\right)-u_{i}\left(x_{\alpha}\right),
$$

then from (5.2), we have:

$$
-2 \epsilon+u_{i}^{m}(x)-u_{i}^{m}\left(x_{\alpha}\right) \leq u_{i}(x)-u_{i}\left(x_{\alpha}\right) \leq 2 \epsilon+u_{i}^{m}(x)-u_{i}^{m}\left(x_{\alpha}\right) .
$$

Passing to limit with respect to $\alpha$ in "Eq.(5.4)" and taking $\epsilon \rightarrow 0$, we get :

$$
\begin{aligned}
& \lim _{\alpha} \sup \left(u_{i}^{m}(x)-u_{i}^{m}\left(x_{\alpha}\right)\right) \leq \lim _{\alpha} \sup \left(u_{i}(x)-u_{i}\left(x_{\alpha}\right)\right) \leq \\
& \lim _{\alpha} \sup \left(u_{i}^{m}(x)-u_{i}^{m}\left(x_{\alpha}\right)\right) .
\end{aligned}
$$

Since $u_{i}^{m}$ is upper semicontinuous, we have:

$$
u_{i}^{m}(x)-\limsup _{\alpha} u_{i}^{m}\left(x_{\alpha}\right)=0 .
$$

We obtain :

$$
u_{i}(x)-\lim _{\alpha} \sup u_{i}\left(x_{\alpha}\right)=0 .
$$

Thus, $u_{i}$ is upper semicontinuous. Similarly, we can prove that

$$
u_{i}(x)-\lim _{\alpha} \inf u_{i}\left(x_{\alpha}\right)=0
$$

and $u_{i}$ is lower semicontinuous. 


\section{REFERENCES}

Abalo, K.Y. and Kostreva, M.M [2004], Nash and Berge equilibria, Applied Mathematical Letters, Vol. 17, 569-573.

Abalo, K.Y. and Kostreva, M.M [2005], Berge's equilibrium: some recent results from fixed-point theorems, Applied Mathematics and Computation, Vol. 169, 624-638.

Aliprantis, C.D. and Border, K.C [1999], Infinite Dimentional Analysis, 2nd edition, Springer, Berlin, New York.

Aubin, J.P. [1984], Mathematical Methods of Game and Economic Theory, North Holland, Amsterdam, New york.

Aumann, R. [1959], Acceptable Points in a General Cooperative n-Person Games in Contribution to the Theory of Games, Vol. 4, Princeton University Press.

Berge, C. [1957], Théorie Générale des Jeux à $n$ Personnes, Gautier Villars, Paris.

Borglin, A. and Keiding, H. [1976], Existence of equilibrium actions and of equilibrium, $J$. Math.Econom, Vol. 3, 313-316.

Browder, F.E [1968], The fixed point theory of multivalued mappings in topological vector spaces, Math.Ann, Vol. 177, 283-301.

Engelking, R. [1968], Outline of General Topology, Institute of Mathematics of the Polish Academy of Sciences, North Holland Publishing Company- Amsterdam.

Fan, K. [1972], A Minimax Inequality and Applications, in Inequalities III, O Shisha ed., Academic Press, New York, London.

Florenzano, M. [2003], General Equilibrium Analysis- Existence and Optimality Properties of Equilibrium, Kluwer, Boston, Dordrecht, London.

Klein,E. and Thompson, A. [1984], Theory of Correspondences, Wiley, New York.

Krim, F. [2001], Etude Approfondie de l'Équilibre de Berge, Magister Thesis, University of Mouloud Mammeri of Tizi-Ouzou, Tizi-Ouzou, Algeria.

Larbani, M. and Nessah, R. [2001], Sur l'équilibre fort selon Berge, RAIRO, Vol. 35, 439-451.

Larbani, M. and Nessah, R. [2008], A note on the existence of Berge and Berge-Nash equilibria, Mathematical Social Sciences, Vol. 55, 258-271.

Larbani, M., Nessah, R. and Tazdait.T [2007], A note on Berge equilibrium, Applied Mathematics Letters, Vol. 20, 926-932.

Myerson, R.B. [1978], Refinements of the Nash equilibrium concept, International Journal of Game Theory, Vol. 7, 73-80.

Nash, J.F. [1951], Non cooperatives games, Ann Maths, Vol. 54, 286-295.

Selten, R. [1975], Reexamination of the perfectness concept for equilibrium point in extensive games, International Journal of Game Theory, Vol. 4, 25-55.

Wu, W.T. and Jiang, J.H. [1962], Essential equilibrium point of n-person noncooperative games, Sci.Sinica, Vol. 11, 1307-1322.

Yu, J. [1999], Essential equilibrium of n-person noncooperative games, Journal of Mathematical Economics, Vol. 31, 361-372.

Zhukovskii, V.I. [1994], Linear Quadratic Differential Games, Naoukova Doumka, Kiev.

Zhou, YH., Yu, J. and Xiang, SW. [2007], Essential stability in games with infinitely many pure strategies, International Journal of Game Theory, Vol. 35, 493-503.

Aknowledgements : The authors would like to thank the anonymous referees for their helpful remarks and comments. 